\title{
Propuesta de atención a alumnos con niveles de depresión en el nivel superior, en el estado de Yucatán
}

\section{Proposal of attention to students with levels of depression at the higher level, in the state of Yucatán}

PEREZ-GARMENDIA, Gloria†*, PEREZ-PEREZ, Iris Cristel, LOPEZ-VALDIVIESO, Leticia y BALDERRABANO-BRIONES, Jazmín

\author{
Tecnológico Nacional de México, Campus Mérida, Campus Villahermosa y Campus Úrsulo Galván \\ ID $1^{\text {er }}$ Autor: Gloria, Perez-Garmendia / ORC ID: 0000-0002-1215-0175, Researcher ID Thomson: G-3863-2018, CVU \\ CONACYT ID: 291627 \\ ID $1^{\text {er }}$ Coautor: Iris Cristel, Perez-Perez / ORC ID: 0000-0003-3120-5597, Researcher ID Thomson: G-1891-2018, CVU \\ CONACYT ID: 843577
}

ID $2^{\text {do }}$ Coautor: Leticia, Lopez-Valdivieso / ORC ID: 0000-0001-6288-3636, Researcher ID Thomson: G-5753-2018, CVU CONACYT ID: 67839

ID $3^{\text {er }}$ Coautor: Jazmín, Balderrabano-Briones / ORC ID: 0000-0002-2925-3234, Researcher ID Thomson: G-3202-2018

DOI: $10.35429 / J E S C .2020 .12 .4 .20 .27$

Recibido: 14 de Octubre, 2020; Aceptado 07 de Diciembre, 2020

\begin{abstract}
Resumen
Objetivos: Proponer estrategias de atención a alumnos con niveles de trastornos de depresión en los alumnos de nivel superior a través de la medición de los síntomas de depresión en estudiantes de nivel superior y con la participación de expertos en el área psicosocial proponer medidas de apoyo del modelo cognitivo. Metodología: La investigación es de tipo exploratoria y descriptiva y diseño no experimental transversal (Hernández, et al, 2006), iniciando con una investigación documental, posteriormente medir el nivel de depresión a través de instrumentos que detecta la presencia de la depresión en los estudiantes para generar la propuesta de atención y estrategias de acuerdo a las causas más fuertes para atender a los alumnos con niveles de trastornos de depresión. Contribución: En el semestre enero junio 2020, el total de alumnos inscritos fueron 903 alumnos. Del total de alumnos inscritos, 473 alumnos, (un 53\%) respondieron el instrumento de medición de depresión, es importante destacar la participación comprometida de los líderes y expertos en el análisis de la información para la generación de una propuesta integral para la atención de los alumnos de nivel superior, en el Estado de Yucatán.
\end{abstract}

Depresión, Ansiedad, Entorno

\begin{abstract}
Objectives: Propose care strategies for students with levels of depression disorders in higher level students through the measurement of depression symptoms in higher level students and with the participation of experts in the psychosocial area, propose support measures of the cognitive model. Methodology: The research is of an exploratory and descriptive type and a non-experimental cross-sectional design (Hernández, et al, 2006), beginning with a documentary research, subsequently measuring the level of depression through instruments that detect the presence of depression in students to generate the proposal of attention and strategies according to the strongest causes to attend to students with levels of depression disorders. Contribution: In the January-June 2020 semester, the total number of registered students was 903 students. Of the total number of enrolled students, 473 students $(53 \%)$ responded to the depression measurement instrument, it is important to highlight the committed participation of leaders and experts in the analysis of information for the generation of a comprehensive proposal for the care of higher level students, in the State of Yucatan
\end{abstract}

Depression, Anxiety, Environment

Citación: PEREZ-GARMENDIA, Gloria, PEREZ-PEREZ, Iris Cristel, LOPEZ-VALDIVIESO, Leticia y BALDERRABANO-BRIONES, Jazmín. Propuesta de atención a alumnos con niveles de depresión en el nivel superior, en el estado de Yucatán. Revista Ciencias de la Educación. 2020. 4-12: 21-28

\footnotetext{
* Correspondencia del Autor: (Correo electrónico: gloriaperez22@ hotmail.com)

$\dagger$ Investigador contribuyendo como primer autor.
} 


\section{Introducción}

\section{¿Qué es el estrés?}

El Dr, Hans Selye en 1926 lo definió como la respuesta adaptativa del organismo ante los diversos estresores. Lo denominó "Síndrome general de adaptación”. En 1976, McGrath lo conceptualiza como un desequilibrio entre las demandas ambientales y la capacidad de respuesta orgánica, señala que el concepto de estrés resulta complejo y ambiguo, aunque hace referencia a una realidad física, psicológica y social importante en la vida moderna. "Es un desequilibrio sustancial (percibido) entre la demanda y la capacidad de respuesta (del individuo) bajo condiciones en las que el fracaso antes demanda posee importantes consecuencias percibidas" en Estremera A., (2017).

Gan, F. y Triginé, J. (2012). Lo pone en el plano de la presión, de la crisis de las respuestas fisiológicas de nuestro organismo que pueden llegar a ser psicosomáticas y con ello afectar la relación mente-cuerpo. La percepción de control que tenemos del agente que produce el estrés es fundamental en el impacto del estrés sobre nuestra conducta y nuestra fisiología. El elemento de control y de predictibilidad determina la magnitud de la respuesta de estrés y la susceptibilidad que este estrés pueda generar secuelas conductuales, cognitivas y fisiológicos sobre el individuo. Para que el estrés tenga consecuencias negativas sobre la salud, la experiencia tendrá que ser percibida como aversiva. Redolar, D. (2015. P.109). Que se puede concretar en la respuesta que tiene el organismo ante las percepciones de incertidumbre.

\section{La ansiedad y la depresión.}

Las emociones negativas no llegan en estado puro casi nunca. De hecho, la ansiedad y la depresión suelen ir juntas. Sin embargo, estos sentimientos son muy distintos el uno del otro. La ansiedad es consecuencia de la percepción de peligro. No se puede sentir angustiado si no se dice a sí mismo que va a pasar algo terrible.

Si está deprimido, es casi seguro que se sentirá angustiado. Y si está luchando contra la ansiedad, es posible que también se sienta deprimido. Burns, D. (2006).
Ahondando en estos sentimientos, la ansiedad puede identificarse como una molestia que puede llegar a incapacitarle e impedirle hacer lo que quiere hacer de verdad con su vida. (Burns, 2006). La ansiedad adopta muchas formas distintas:

Preocupación crónica. Se preocupa constantemente por su familia, su salud, su carrera profesional o su economía. Se le revuelve el estómago y tiene la impresión de que está a punto de pasarle algo malo, aunque no es capaz de determinar cuál es exactamente el problema. Miedos y fobias. Puede tener miedo a las agujas, a la sangre, a las alturas, a los ascensores, a conducir, a viajar en avión, al agua, a las arañas, a las serpientes, a los perros, a las tormentas, a los puentes o a quedarse atrapado en lugares cerrados.

Ansiedad por la actuación. Se queda paralizado siempre que tiene que hacer un examen, actual o hacer algo delante de otras personas o competir en una prueba deportiva.

Ansiedad por hablar en público. Se pone nervioso cuando tiene que hablar ante un grupo porque se dice a si mismo: "temblaré y todos verán lo nervioso que estoy. Se me quedará la mente en blanco y haré el idiota. Todos me despreciarán y me tomarán por un neurótico total",

Timidez. Se siente nervioso y apurado en las reuniones sociales se dice a sí mismo: "todo el mundo parece encantado y relajado. Pero yo no tengo nada interesante que decir. Lo más probable es que se den cuenta de lo tímido que soy de lo fuera de lugar que me siento. Deben de pensarse que soy una especie de tipo raro o de fracasado. Soy el único que se siente así. ¿Qué me pasa?"

Ataques de pánico. Se siente ataques de pánico repentinos, terribles, que parece como si aparecieran sin más y atacaran de manera inesperada, como un rayo. En los ataques se siente mareado, el corazón le palpita con fuerza y se tiene hormigueos en los dedos. Quizá se piense "debo de estar teniendo un ataque al corazón. ¿Y si me desmayo, o me muero? ¡no puedo respirar! ¿y si me ahogo?". Se intenta aferrar a la vida. Al poco rato, la sensación de pánico desaparece de manera tan misteriosa como llegó, dejando una sensación de desconcertación, susto y humillación. 
Se pregunta qué habrá pasado y cuándo volverá a pasarle aquello.

Agorafobia. Es el miedo a estar fuera de casa, porque se piensa que va a pasar algo terrible (que va a sufrir un ataque de pánico, quizás) y que no habrá nadie que pueda ayudar. Puede ser miedo a los espacios abiertos, a los puentes, a las multitudes, a estar en la cola del supermercado o a viajar en transportes público.

Obsesiones y compulsiones: Es el acosamiento de pensamientos obsesivos que no pueden quitarse de la mente e impulsos compulsivos de realizar rituales supersticiosos para controlar sus miedos. Por ejemplo: el miedo a los microbios y tener el impulso irresistible de lavarse las manos una y otra vez, todo el día.

Trastorno de estrés postraumático: El acosamiento de los recuerdos o imágenes de algún suceso terrible que sucedió hace meses, e incluso hace años, como una violación, unos malos tratos, torturas o asesinatos.

Preocupación por su aspecto físico (trastorno dismórfico corporal): La sensación de que el aspecto tiene algo de grotesco o de anormal a pesar de que sus amigos y su familia le intentan tranquilizar diciéndole que tiene un aspecto normal. Puede ser el pensamiento de una nariz deformada, que el cuerpo tiene una forma rara. Se puede pasar muchísimo tiempo consultando a cirujanos estéticos.

Preocupaciones por su salud (hipocondría): Va de médico en médico quejándose de molestias, dolores, fatiga, mareos $\mathrm{u}$ otros síntomas. Es el estar seguro que se padece alguna enfermedad terrible, pero el médico siempre le tranquiliza diciéndole que no le pasa absolutamente nada. Se siente aliviado durante algunos días, pero no tarda en empezar a obsesionarse de nuevo con la salud.

\section{Metodología}

El objetivo general de la investigación fue proponer estrategias de atención a alumnos con niveles de trastornos de depresión en los alumnos de educación superior en Mérida Yucatán, primero fue medir los estados de ánimo con el que se detecta la presencia de la depresión y se mide su gravedad.

\section{Desarrollo metodológico}

Se realizó una investigación de tipo exploratoria y descriptiva y diseño no experimental transversal (Hernández, et al, 2006), primero se desarrolla una investigación documental de las investigaciones realizadas, posteriormente se mide el nivel de depresión a través de instrumentos que miden los estados de ánimo con el que se detecta la presencia de la depresión que ocasionan las disfunciones para posteriormente hacer las propuestas hechas en contexto semejantes de la población a investigar.

El método fue el estudio de campo con las técnicas de encuesta a través de formularios en https://docs.google.com/forms, para medir los síntomas de la depresión de Beck@1987 con 21 preguntas de opción múltiple, cuyas respuestas van desde cero hasta tres en la frecuencia que el sujeto ha experimentado en síntomas como como tristeza, desanimo, culpabilidad, suicidio, irritabilidad, perdida de interés, seguridad e insomnio. Se aplicaron del 12 de febrero al 12 de marzo de 2020 a los alumnos inscritos en el semestre enero junio 2020. Con los resultados obtenidos se identificarán estrategias de acuerdo a las causas más fuertes para atender a los alumnos con niveles de trastornos de depresión.

\section{Sujetos de estudio}

La población a medir fueron 903 alumnos inscritos en el semestre enero junio 2020 de los cuales 488 alumnos inscritos de L.A y 415 alumnos inscritos en I.G.E. Se les aplicó el cuestionario través de la plataforma de Microsoft, visitando cada uno de los grupos abiertos en el semestre enero junio 2020. El instrumento fue aplicado del 12 de febrero al 12 de marzo de 2020.

\section{Desarrollo de instrumentos}

El instrumento de medición (apéndice A) Inventario de la depresión, de Beck, copyrigh 1978, Dr. Aaron T. Beck, mide la frecuencia que el sujeto ha experimentado en síntomas como tristeza, desanimo, culpabilidad, suicidio, irritabilidad, perdida de interés, seguridad e insomnio. Consta de 21 ítems, con cuatro opciones de respuesta, con 0 (grado mínimo a 3 (grado máximo) el total más alto posible de todo el cuestionario es sesenta y tres. Según la guía para la interpretación del cuestionario de la depresión, (tabla 1) de Beck, 2012. 
Una puntuación persistente de 17 o más indica que puede necesitar ayuda profesional.

\begin{tabular}{|l|l|}
\multicolumn{1}{|c|}{$\begin{array}{c}\text { Puntuación } \\
\text { total }\end{array}$} & \multicolumn{1}{|c|}{ Niveles de depresión. } \\
\hline $1-10$ & $\begin{array}{l}\text { Estos altibajos son considerados } \\
\text { normales. } \\
\text { Leve perturbación del estado de } \\
\text { ánimo. }\end{array}$ \\
$11-16$ & $\begin{array}{l}\text { Estados de depresión intermitentes. } \\
\text { Depresión moderada. } \\
17-20\end{array}$ \\
$21-30$ & Depresión grave \\
$31-40$ & Depresión extrema. \\
Más de 40 &
\end{tabular}

Tabla 1 Guía de interpretación del cuestionario de la depresión de Beck copyright, 1978

Fuente: Beck, 2012, Sentirse bien, una nueva terapia contra las depresiones. Pp.39

El instrumento se aplicó en el mes de febrero de 2020. El método fue el estudio de campo con las técnicas de encuesta a través de formularios en https://docs.google.com/forms, cabe hacer la observación que se aplicó antes que iniciara la contingencia del Covid 19, que afecta actualmente el mundo.

\section{Resultados}

\section{Resultados del instrumento de medición de depresión en alumnos inscritos periodo enero-junio 2020}

En el semestre enero junio 2020, el total de alumnos inscritos fueron 903 alumnos. Del total de alumnos inscritos, 473 alumnos, (un 53\%) respondieron el instrumento de medición de depresión, de los cuales un $54 \%$ de los alumnos, fueron mujeres y un $46 \%$ de hombres. (Tabla 3.) Del total de alumnos que respondieron el instrumento de medición de depresión, (Tabla 4) un 64. \% de los alumnos fueron de L.A. y un 36 $\%$ de IGE.

Los resultados encontrados en el instrumento de medición de depresión en los alumnos inscritos y de acuerdo a la guía para la interpretación del cuestionario de la depresión, de Beck, 2010. Una puntuación persistente de 17 o más indica que puede necesitar ayuda profesional. Según los resultados de los sujetos de investigación, existe un $17.1 \%$ de alumnos que están en este nivel y que necesitan ayuda profesional. En un nivel que identifica como leve perturbación del estado de ánimo esta un $17.3 \%$ de alumnos que están en este nivel. (Tabla 1.)

\begin{tabular}{|l|l|r|r|}
\hline \multicolumn{1}{|c|}{ Nivel } & \multicolumn{1}{c}{ Definición } & \multicolumn{1}{c|}{ Alumnos } & \multicolumn{1}{c|}{$\%$} \\
\hline $1-10$ & $\begin{array}{l}\text { Son considerados } \\
\text { normales }\end{array}$ & 310 & 65.5 \\
\hline $11-16$ & $\begin{array}{l}\text { Leve perturbación del } \\
\text { estado de ánimo }\end{array}$ & 82 & 17.3 \\
\hline $17-20$ & $\begin{array}{l}\text { Estados de depresión } \\
\text { intermitentes }\end{array}$ & 28 & 5.9 \\
\hline $21-30$ & Depresión moderada & 42 & 8.9 \\
\hline $31-40$ & Depresión grave & 8 & 1.7 \\
\hline más de 40 & Depresión extrema & 3 & 0.6 \\
\hline & Total entrevistados & $\mathbf{4 7 3}$ & $\mathbf{1 0 0 . 0}$ \\
\hline
\end{tabular}

Tabla 2. Resultado del instrumento de depresión en alumnos inscritos periodo enero-junio 2020

Fuente: Elaboracón Propia

En la tabla 3, se identifica que existe una mayor incidencia en las mujeres que se encuentran con más de 17 puntos en los resultados de niveles de depresión, con un $22.76 \%$ y con solo un $10.26 \%$ de los hombres. Así también se identifica que por cada 7 mujeres que tienen depresión grave, solo 1 hombre lo tiene.

Así también se muestra que de los sujetos encuestados un $58.30 \%$ de las mujeres están en el rango "de considerados normales" y en mayor porcentaje se encuentran los hombres en este mismo nivel con un $74.76 \%$.

\begin{tabular}{|l|l|r|r|r|r|}
\hline \multicolumn{1}{|c}{ Nivel } & \multicolumn{1}{c|}{ Definición } & Hombres & \multicolumn{3}{c|}{ Mujeres } \\
\hline $1-10$ & Son considerados normales & 159 & 74.76 & 151 & 58.30 \\
\hline $11-16$ & $\begin{array}{l}\text { Leve perturbación del estado } \\
\text { de ánimo }\end{array}$ & 33 & 15.42 & 49 & 18.91 \\
\hline $17-20$ & $\begin{array}{l}\text { Estados de depresión } \\
\text { intermitentes }\end{array}$ & 9 & 4.20 & 19 & 7.33 \\
\hline $21-30$ & Depresión moderada & 12 & 5.60 & 30 & 11.58 \\
\hline $31-40$ & Depresión grave & 1 & .46 & 7 & 2.70 \\
\hline más de 40 & Depresión extrema & 0 & & 3 & 1.15 \\
\hline & Total entrevistados & $\mathbf{2 1 4}$ & & $\mathbf{2 5 9}$ & \\
\hline
\end{tabular}

Tabla 3 Resultado del nivel de depresión por género en alumnos inscritos en el periodo Enero-Junio 2020

Fuente: Elaboracón Propia

En la tabla 4, se identifica que existe una mayor incidencia en la carrera de IGE que se encuentran con más de 17 puntos en los resultados de niveles de depresión, con un $23.96 \%$ y con solo un $13.23 \%$ de la carrera de LA. Así también se identifica que por cada 2 alumnos de la carrera de LA que tienen depresión grave, solo 1 de la carrera de IGE lo tiene.

Haciendo una separación por carreras, se puede notar que la carrera de LA tiene un $70.86 \%$ de sujetos que están en el nivel "son considerados normales" y con un 56.14 en los de la carrera de IGE. 


\begin{tabular}{|l|l|r|r|r|r|}
\hline \multicolumn{1}{|c}{ Nivel } & \multicolumn{1}{c}{ Definición } & \multicolumn{1}{c|}{ IGE } & \multicolumn{1}{c|}{ \%A } \\
\hline $1-10$ & $\begin{array}{l}\text { Son considerados } \\
\text { normales }\end{array}$ & 96 & 56.14 & 214 & 70.86 \\
\hline $11-16$ & $\begin{array}{l}\text { Leve perturbación del } \\
\text { estado de ánimo }\end{array}$ & 34 & 19.88 & 48 & 15.89 \\
\hline $17-20$ & $\begin{array}{l}\text { Estados de depresión } \\
\text { intermitentes }\end{array}$ & 9 & 5.26 & 19 & 6.29 \\
\hline $21-30$ & Depresión moderada & 28 & 16.37 & 14 & 4.63 \\
\hline $31-40$ & Depresión grave & 3 & 1.75 & 5 & 1.65 \\
\hline más de 40 & Depresión extrema & 1 & .58 & 2 & .66 \\
\hline & Total entrevistados & $\mathbf{1 7 1}$ & & $\mathbf{3 0 2}$ & \\
\hline
\end{tabular}

Tabla 4 Resultado del nivel de depresión por carrera en alumnos inscritos en el periodo Enero-Junio 2020 Fuente: Elaboracón Propia

Haciendo una síntesis de los resultados de niveles de depresión en los sujetos encuestados existe un $17.1 \%$ que se encuentra en niveles que requieren ayuda profesional, al encontrarse en puntos de 17 o más. Esto quiere decir que de 473 alumnos encuestados 81 sujetos requieren ayuda profesional. En un grado menor de depresión se encuentra un $17.3 \%$ que se encuentran en una leve perturbación del estado de ánimo con nivel de 11 a 16 puntos. Y con un resultado mucho más fuerte se encuentran un 65 $\%$ que son considerados normales, con un nivel de 1-10 puntos.

\section{Conclusiones}

Los resultados encontrados (Tabla 5) con niveles por arriba de 17 puntos, indican que pueden necesitar ayuda profesional, (Burns, 2012), representan el $17.1 \%$ de los sujetos encuestados. Se hace la observación que la aplicación de los instrumentos fue con motivos estadísticos, y no se pidieron los nombres de los sujetos. Esta investigación tiene como objetivo dar propuestas que apoyen, primero a identificar a los alumnos que estén en niveles altos de depresión y poder darles una herramienta para que puedan registrar diariamente su estado de ánimo (apéndice B), y en el formato de autoayuda, la terapia cognitivoconductual (TCC), donde se aprende a cambiar los pensamientos negativos, o "cogniciones", que provocan la depresión, así como las pautas de conducta contraproducentes que mantienen "atascado". (Burns, 2012)

\begin{tabular}{|l|l|r|r|}
\hline \multicolumn{1}{|c|}{ Nivel } & \multicolumn{1}{c|}{ Definición } & Alumnos & \multicolumn{1}{c|}{$\%$} \\
\hline $1-10$ & Son considerados normales & 310 & 65.5 \\
\hline $11-16$ & $\begin{array}{l}\text { Leve perturbación del estado de } \\
\text { ánimo }\end{array}$ & 82 & 17.3 \\
\hline $17-20$ & Estados de depresión intermitentes & 28 & 5.9 \\
\hline $21-30$ & Depresión moderada & 42 & 8.9 \\
\hline $31-40$ & Depresión grave & 8 & 1.7 \\
\hline más de 40 & Depresión extrema & 3 & 0.6 \\
\hline & Total entrevistados & $\mathbf{4 7 3}$ & $\mathbf{1 0 0 . 0}$ \\
\hline
\end{tabular}

Tabla 5 Resultado del instrumento de depresión en alumnos inscritos periodo enero-junio 2020.

Fuente: Elaboracón Propia

ISSN: 2523-2436

ECORFAN® Todos los derechos reservados

\section{Propuesta}

Parte del objetivo de esta investigación es hacer propuestas para atender a los alumnos con niveles de trastornos de depresión, para lo cual se proponen los expertos en estos temas como Burns, 2012. Esta propuesta se basa en investigaciones que el Dr. D. Burns ha realizado a lo largo de su vida en la psiquiatría, donde propone que la "biblioterapia" basada en la lectura de Sentirse bien (libro que el autor publicó) debería ser la primera línea de tratamiento para la mayoría de los pacientes que padecen depresión, ya que suele dar resultados más rápidos que los medicamentos o que la psicoterapia. Los estudios señalados del Dr. Scogin (1987) en Burns, 2012, demuestran que la (TCC) Terapia cognitivo-conductual, puede resultar muy eficaz para muchos individuos en un formato de autoayuda, incluso sin tomar pastillas ni recibir la orientación de un terapeuta. Primero se requiere una autoevaluación del estado de ánimo (apéndice B), que, de acuerdo a Burns, se debe hacer las veces que así lo decida o por lo menos una vez a la semana.

La autoevaluación consta de cuatro apartados: Sentimientos de ansiedad, sentimiento de ansiedad física, depresión e impulsos suicidas. Los apartados para interpretar la puntuación se encuentran en el apéndice.

Las investigaciones indican que muchos pacientes que sufren ansiedad pueden superar el problema por su cuenta, sirviéndose de herramientas cognitivas.Las puntuaciones de la autoevaluación pueden cambiar rápidamente. Estar absolutamente preocupado y estresado un día, y mucho más confiado y relajado al día siguiente. Además, la ansiedad depende mucho de la situación en que se encuentre. Si es tímido, pero evita tratar con la gente, es probable que no llegue a sentir mucha ansiedad.

El registro diario de pensamientos negativos de Burns es el inicio de 5 pasos que propone para la autoayuda para tratar la depresión, es posible que en esta sección la redacción sea en segunda persona, debido a que está dirigida a la persona que necesite ayuda: 


\section{Paso 1. Suceso trastornador}

Al principio del registro diario de estado de ánimo escriba una breve descripción de un momento en que usted se siente trastornado. Asegúrese de que el problema que describe es concreto en cuanto a persona, lugar y tiempo. Puede tratarse cualquier momento en que se haya sentido decaido, preocupado o con pánico. Unas preguntas oportunas que se puede hacer son las siguientes:

\section{- $\quad$ ¿Qué hora del día era? \\ - ¿Dónde estaba yo? \\ - ¿Qué estaba pasando?}

Todos los problemas quedarán fijados en un momento determinado en el que el sujeto estaba sufriendo. Resultará mucho más sencilla la tarea si registra los pensamientos y los sentimientos negativos sobre el papel. Así podrá centrarse en ellos de uno en uno. (la terapia a base de hablar de manera no estructurada tiene la tendencia a alargarse de forma interminable sin que nada cambie verdaderamente). Para conseguir bajar los sentimientos de ansiedad, de inseguridad o de depresión, tendrá que tomar el registro diario de estado de ánimo y ponerse a trabajar en ello. Tiene que centrarse en un momento concreto en que se sintió trastornado. En ese momento único estará fijada la clave de todos sus sentimientos.

\section{Paso 2. Emociones.}

Cuando haya descrito el suceso trastornador, rodee con un círculo todas las palabras que describan cómo se siente y puntúe cada sentimiento en una escala del $0 \%$ (nada en absoluto) al $100 \%$ (extremadamente). Lleve sus puntuaciones a la columna de "\% antes". Cuando haya completado el registro diario de estado de ánimo, podrá puntuar de nuevo sus sentimientos en la columna de "\% antes"

Es importante que identifique y puntúe sus emociones negativas porque tipos concretos de sentimientos son consecuencia de tipos determinados de pensamientos negativos. Al hacer esto le resultará más fácil después identificar sus pensamientos negativos en el paso 3. Las emociones que se relacionan en el registro diario de estado de ánimo son las siguientes:
Ansiedad, nervios o preocupación: usted se dice a sí mismo que corre peligro y que está a punto de suceder algo terrible.

- Pánico: se dice a sí mismo que está a punto de morirse, de ahogarse, de desmayarse, de perder el control o de volverse loco.

- Turbación: se dice que ha quedado tot;into delante de otras personas.

- $\quad$ Timidez: Usted se dice que las demás personas verán lo nervioso e inseguro que se siente y le despreciarán.

- $\quad$ Soledad: Se dice que no le puede querer nadie y que está condenado a quedarse solo para siempre.

- Depresión: se dice que es un fracaso o que ha perdido algo que es importante para su sentido de la autoestima.

- Desesperanza: se dice que sus problemas no se resolverán nunca y que sus sufrimientos serán para siempre.

- Culpa: se dice que es una mala persona o que ha quebrantado su propio sistema de valores.

- Vergüenza: se dice que las demás personas verán lo malo, lo imperfecto o lo defectuoso que es usted y le despreciarán.

- Inferioridad: se dice que no es tan bueno como las demás personas o que no es bueno como debería ser.

- Frustración: se dice que las demás personas, o lo hechos, deberían ser como usted espera que sean.

- Ira: se dice que los demás son unos canallas egoístas que le están tratando injustamente $\mathrm{o}$ que se estan aprovechando de usted intencionadamente.

Sentirse atrapado: Se dice que tiene que ceder ante las exigencias de su cónyuge, de su novio o novia, de sus amigos o de su familia.

\section{Paso 3. Pensamientos negativos}

Anote los pensimientos negativos que le pasan por la mente cuando se siente trastornado. Formúlese preguntas como la siguiente: "Cuando me siento desesperanzado y desanimado. ¿qué me estoy diciendo a mi mismo?. " "Cuando me siento lleno de pánico, ¿Qué mensajes me estoy transmitiendo a mi mismo?" 
Enumérelos en la columna de "pensamientos negativos" de su registro diario de estado de ánimo y califique el grado en que usted cree cada uno de los pensamientos, desde el $0 \%$ (nada en absoluto) hasta el $100 \%$ (completamente). Anote sus puntuaciones en la columna de \% antes. He aquí algunos consejos que deberá tener presentes al complementar el paso de los pensamientos negativos:

Escriba frases completas. No escriba "volverme loco" en la columna de "pensamientos negativos" porque eso no significa nada. En vez de ello, puede escribir: "estoy a punto de volverme loco".

- $\quad$ Limite cada pensamiento negativo a una extensión razonable. Con una sola frase suele bastar. Es dificil trabajar con pensamientos largos y farragosos.

Procure evitar las preguntas retóricas tales como "¿Por que estoy tan angustiado siempre?" o "qué me pasa?". No debe hacerlo así porque una pregunta no se puede desmentir. Sin embargo, una pregunta retórica se puede convertir facilmente en un pensamiento negativo, por ejemplo: "no debería estar siempre tan angustiado" o " debe de pasarme algo".

No ponga descripciones de sus sentimientos en la columna de "pensamientos negativos". Evite afirmaciones tales como : "estoy preocupado por mi examen o "siento pánico ahora mismo". Anote sus sentimientos en la sección de "emociones" del registro diario de estado de ánimo. Si describe sus sentimientos en la columna de "pensamientos negativos", se sentirá frustrado porque los sentimientos no se pueden rebatir. Sin embargo, sí que se pueden desmentir los pensamientos destorsionados que provocan el sentimiento negativo.
$-$

Asegúrese de que el pensamiento negativo no incluye la descripción de un suceso. Por ejemplo, "Teresa me rechazó y me siento desgraciado" no debe estar en la columna de "pensamientos negativos" porque se trata de la descripción de un suceso (que Teresa me rechazó) y de un sentimiento (me siento desgraciado). Anote el suceso en la parte superior del registro diario de estado de ánimo y enumere justo debajo sus emociones (solitario, dolido, enfadado, inferior, rechazado, desanimado y desgraciado). En la columna de "pensamientos negativos" puede anotar los pensamientos que provocaron estos sentimientos. Como por ejemplo:

1. "Todo es culpa mía." $100 \%$

2. "Ningun hombre me querrá nunca." $100 \%$

3. "siempre estaré solo." $100 \%$

\section{Paso 4. Distorsiones}

Anote las distorciones de cada pensamiento negativo en la columna de "distorsiones". En general encontrará muchas distorsiones en cada pensamiento. Puede enumerar las distorsiones en su registro diario de estado de ánimo puede servirse de abreviaturas para ahorrar tiempo. Por ejemplo, puede escribir MAG como abreviatura de "magnificación". PE como abreviatura de "poner etiquetas". ATD como abreviatura de "afirmaciones del tipo [deberia]" y AI como abreviatura de "autoinculpación".

\section{Paso 5. Pensamentos positivos.}

Genere más pensamentos positivos y realistas que desmientan sus pensamientos negativos. Preguntese a sí mismo: “¿Puedo pensar en este problema de una manera más positiva y realista?". Recuerde las condiciones necesaria y suficiente para el cambio emocional.

\footnotetext{
- La condición necesaria: el pensamientopositivo deber ser verdadero al cien por cien.

- $\quad$ La condición sufiente: el pensamiento positivo debe desmentir el pensamiento negativo.
} 
El paso 5 será dificil. ¡Si desmentir sus pensamiento negativos fuera fácil, a usted no le haria falta este metodo. Indique el grado en que cree cada pensamiento positivo en la columna de "\% creencia" y reevalúe después su creencia en el pensamiento negativo correspondiente.

Es probable que en lo primeros esfuerzos no serán muy eficaces por uno o dos motivos. O bien los pensamientos positivos no serán verdaderos al cien por cien, con lo que no cumplirán la conedición necesaria para el cambio emocional, o bien no desmentirán los pensamientos negativos, por lo que no cumplian la condición suficiente para el cambio emocional. Para esto es es necesario conocer las técnicas de modelo cognitivo de Burns, (2006)

\section{Referencias}

Burns, D. (2012) Sentirse bien, Una nueva terapia contra las depresiones. México: Paidos.

Burns D. (2006). Adiós, ansiedad, como superar la timidez, los miedos, las fobias y las situaciones de pánico. México: Paidos.

Estremera A. (2017). Influencia de las variables socio-demográficas y laborales en los valores de estrés determinados con el modelo desequilibrio esfuerzo recompensa de Siegrist. Tesis Doctoral. TesisA.Estremera-noviembrede2017.pdf

Gan, F. y Triginé, J. (2012). Gestión del estrés. Ediciones Díaz de Santos. https://elibro.net/es/ereader/biblioitmerida/6271 4 ? page $=4$.

Martínez Díaz, E. S., \& Díaz Gómez, D. A. (2007). Una aproximación psicosocial al estrés escolar. Educación y Educadores, 10(2), 11-22. http://www.scielo.org.co/scielo.php

Medina-Mora, M. E.; Borges, G.; Lara Muñoz C.; Benjet C.; Blanco Jaimes J.; Fleiz Bautista, C.; Villatoro Velázquez, J.; Rojas Guiot, E.; Zambrano Ruíz, J.;Casanova Rodas L.; AguilarGaxiola; S. (agosto 2003).Prevalencia de trastornos mentales y uso de servicios: Resultados de la encuesta nacional de epidemiología Psiquiátrica en México. Salud Mental. V. 26 (4).

Redolar, D. (2015). El estrés. Editorial UOC. https://elibro.net/es/ereader/biblioitmerida/5771 $7 ?$
PEREZ-GARMENDIA, Gloria, PEREZ-PEREZ, Iris Cristel, LOPEZVALDIVIESO, Leticia y BALDERRABANO-BRIONES, Jazmín. Propuesta de atención a alumnos con niveles de depresión en el nivel superior, en el estado de Yucatán. Revista Ciencias de la Educación. 2020 